\title{
The immediate impact of the COVID-19 pandemic on motor neuron disease services and mortality in Scotland
}

\author{
Stella A. Glasmacher ${ }^{1,2}$ - Juan Larraz ${ }^{1,2} \cdot$ Arpan R. Mehta $^{1,2,3,4} \cdot$ Patrick K. A. Kearns ${ }^{1,2,3,5} \cdot$ Michael Wong $^{1,2}$. \\ Judith Newton ${ }^{1,2,3} \cdot$ Richard Davenport $^{1,6} \cdot$ George Gorrie $^{7} \cdot$ Ian Morrison ${ }^{8} \cdot$ Javier Carod Artal ${ }^{9}$. \\ Siddharthan Chandran ${ }^{1,2,3,4,6}$. Suvankar Pal ${ }^{1,2,3,6,10}$. CARE-MND Consortium
}

Received: 31 July 2020 / Revised: 28 August 2020 / Accepted: 31 August 2020 / Published online: 5 September 2020

c) Springer-Verlag GmbH Germany, part of Springer Nature 2020

Dear Sirs,

As of August 2020, more than 800,000 deaths worldwide have been attributed to COVID-19, of which $>41,000$ have been in the United Kingdom and 2494 in Scotland [1]. People with MND (pwMND) may be particularly vulnerable. We completed a population-based analysis of the Scottish MND Register, CARE-MND [2] and a clinician survey, to measure the impact of the pandemic on (1) diagnostic rate, (2) mortality rate, and, (3) delivery of services.

We compared all-cause mortality between 01/03-01/06 in 2015-2019 (comparator period) and 01/03-01/06 2020 (COVID-19 period) using multivariable Poisson regression including age $(<50,50-70,>70$ years $)$ and year (2015-2020) as independent variables. The regression coefficients for 2015-2019 were pooled in a generic inverse variance random effects model to yield a summary coefficient. We performed Chi-squared test to compare socioeconomic status (SIMD) [3] between those who died and survivors during the COVID-19 period.

To investigate the impact of the pandemic on care delivery, we undertook a structured online survey of MND

Suvankar Pal

suvankar.pal@ed.ac.uk

1 Anne Rowling Regenerative Neurology Clinic, University of Edinburgh, Chancellor's Building, 49 Little France Crescent, Edinburgh, UK

2 Centre for Clinical Brain Sciences, University of Edinburgh, Chancellor's Building, 49 Little France Crescent, Edinburgh EH16 4SB, UK

3 Euan MacDonald Centre for Motor Neurone Disease Research, University of Edinburgh, Chancellor's Building, 49 Little France Crescent, Edinburgh, UK

4 UK Dementia Research Institute, University of Edinburgh, Chancellor's Building, 49 Little France Crescent, Edinburgh, UK healthcare professionals focussing on their access to diagnostics and interventions; the survey comprised multiple choice and free text questions.

We identified 1062 pwMND, diagnosed between 01/01/2015-01/06/2020 (median age 68 [IQR 60-75], 632 (59.5\%) male). Most (723, 77.7\%) had amyotrophic lateral sclerosis.

The number of new diagnoses remained constant after 2016 (range 42-45). Two pwMND, both with progressive muscular atrophy, died with confirmed or suspected COVID19. One person had an ALSFRS-R 36/48 in July 2020, did not use non-invasive ventilation and died unexpectedly. The other person had an ALSFRS-R of 20/48 in January 2020 and used non-invasive ventilation continuously.

There was no difference in all-cause mortality between the COVID-19 and comparator periods (pooled regression coefficient $1.0995 \%$ CIs $0.78,1.53 ; P=0.61$ ). The mortality rate per 1000 cases was 114 (37/323) in the COVID-19 period and 124 in the comparator period (mean, SD 52.9). Mortality was higher above 70 years age $(1.4895 \%$ CI 1.10 , $1.99 ; P=0.009)$ and lower below 50 years age $(0.4195 \%$ CIs $0.19,0.80 ; P=0.017$, Fig. 1a). There were no differences in

5 MRC Human Genetics Unit, Institute of Genetics and Molecular Medicine, University of Edinburgh, Crewe Road, Edinburgh, UK

6 Department of Clinical Neurosciences, NHS Lothian, Edinburgh, UK

7 Department of Neurology, NHS Greater Glasgow and Clyde, Glasgow, UK

8 Department of Neurology, NHS Tayside, Dundee, UK

9 Department of Neurology, NHS Highland, Inverness, UK

10 Department of Neurology, NHS Forth Valley, Larbert, UK 
(a)

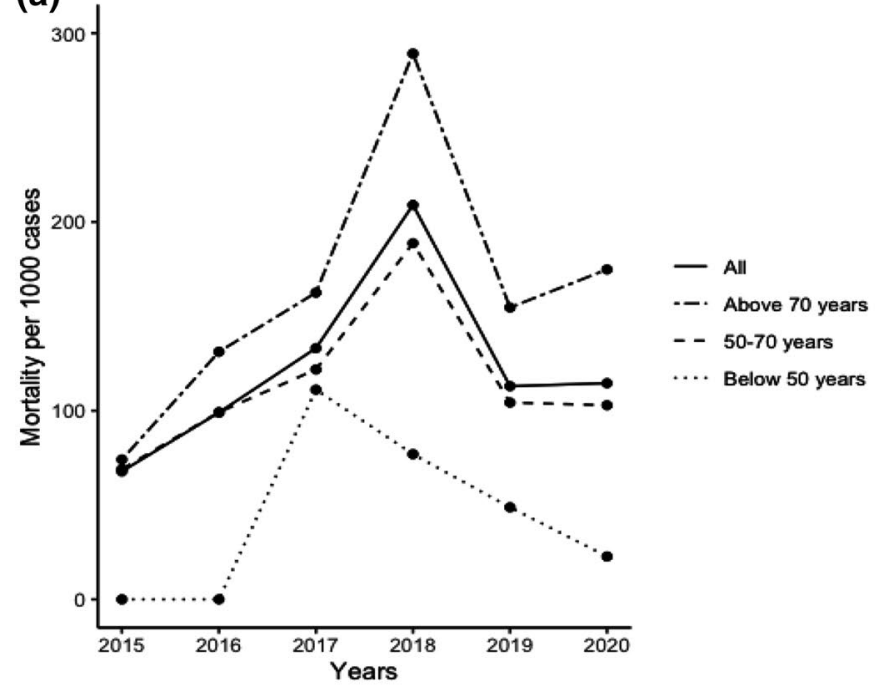

(b)

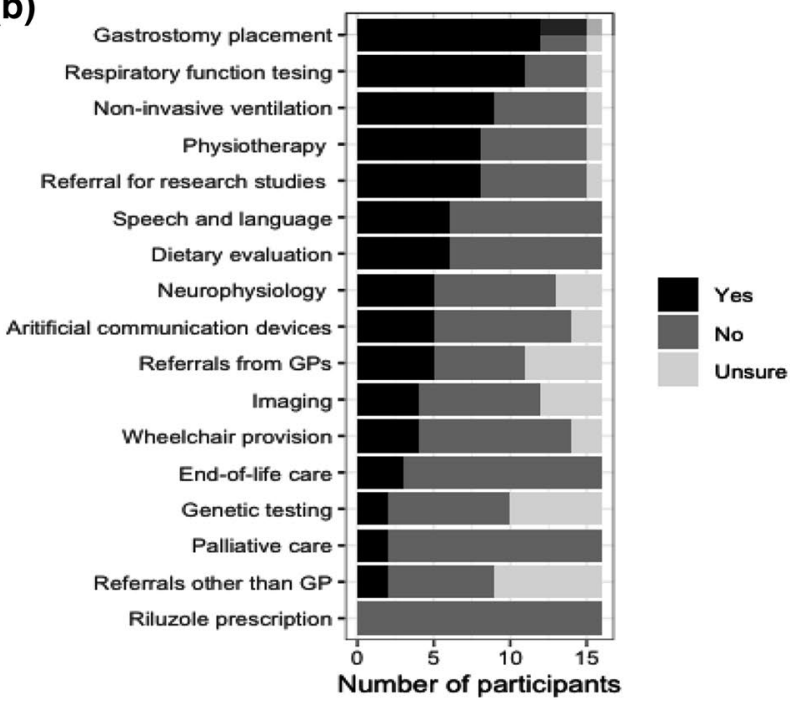

Fig. 1 a Mortality trends according to age group from 01/03-01/06 2015-2020. b Survey respondents' $(n=16)$ answers regarding the clinical services affected during the COVID-19 pandemic

socioeconomic index in those who died compared to survivors (SIMD 1\&2: $38.8 \%$ vs. $34.5 \% ; P=0.42$ ).

Nine consultants and seven nurse specialists completed the survey (representing 13/14 Scottish health boards). The majority reported provision of gastrostomy $(75 \%)$, respiratory function testing $(69 \%)$, and non-invasive ventilation (56\%) were adversely affected. Riluzole prescription (100\%), palliative care (88\%), and end-of-life care (81\%) were comparatively unaffected (Fig. 1b). Most reported reduced ability for face-to-face review. Opinions about whether videoconferencing was an acceptable substitute were divided (strongly agree/agree $43 \%$, strongly disagree/disagree $38 \%$, remainder undecided).

Clinicians additionally reported 70-90\% of pwMND were shielding, and that pwMND expressed heightened anxiety of being exposed to COVID-19, experienced difficulties in receiving support from professional carers and family, felt loneliness, and fears of being denied treatment.

Our study is the first to demonstrate at a national level that rates of new MND diagnoses and all-cause mortality in pwMND have thus far been unaffected by COVID-19. Shielding recommendations by Scottish government may have contributed to the absence of excess mortality. Evaluation of shielding justifies further study including comparison with healthcare systems that did not shield. Comparative international data on mortality and morbidity in pwMND has yet to be published.

Notably, we observed a spike in mortality in 2018 , which may be linked to a higher than usual rate of new diagnoses in 2015.

Our data suggest respiratory function tests, non-invasive ventilation and gastrostomy are the worst affected services.
Preparations for further waves of COVID-19 should prioritize maintenance of these. The adverse impact of COVID19 on longer-term quality of life, carer burden, morbidity and mortality in this vulnerable group requires longitudinal evaluation.

Acknowledgements A.R.M. is a Lady Edith Wolfson Clinical Fellow and is jointly funded by the Medical Research Council and the Motor Neurone Disease Association (MR/R001162/1). S.C.'s group is funded by the UK Dementia Research Institute partner funders: the Medical Research Council, Alzheimer's Research UK and the Alzheimer's Society.

CARE-MND consortium: David Thomson: Department of Neurology, NHS Forth Valley, Larbert, UK. Louise Murrie: Department of Neurology, NHS Fife, Kirkaldy, UK. Jenny Preston: Department of Neurology, NHS Ayrshire \& Arran, Ayr, UK; Department of Neurology, NHS Greater Glasgow \& Clyde, Glasgow, UK. Govind Chavada: Department of Neurology, NHS Ayrshire \& Arran, Ayr, UK; Department of Neurology, NHS Greater Glasgow \& Clyde, Glasgow, UK. Robert Swingler: Centre for Clinical Brain Sciences, Chancellor's Building, University of Edinburgh, 49 Little France Crescent, Edinburgh, UK; Euan MacDonald Centre for Motor Neurone Disease Research, Chancellor's Building, University of Edinburgh, 49 Little France Crescent, Edinburgh, UK. Danielle Leighton: Department of Neurology, NHS Greater Glasgow \& Clyde, Glasgow, UK. Callum Duncan: Department of Neurology, NHS Grampian, Aberdeen, UK. Myles Connor: Department of Neurology, NHS Borders, Melrose, UK. David Simpson: Department of Neurology, NHS Borders, Melrose, UK. Ondrej Dolezal: Department of Neurology, NHS Dumfries and Galloway, Dumfries, UK. Katja Lassak: Department of Neurology, NHS Fife, Kirkaldy, UK. Antonella Benvenga: Department of Neurology, NHS Fife, Kirkaldy, UK. Andrew Bethell: Department of Neurology, NHS Highland, Inverness, UK. Gillian Craig: Department of Neurology, NHS Tayside, Dundee, UK. Laura Cunningham: Department of Neurology, NHS Greater Glasgow \& Clyde, Glasgow, UK. Moira Flett: Department of Neurology, NHS Orkney, Orkney, UK. Janice Hatrick: Department of Neurology, NHS Greater Glasgow \& Clyde, Glasgow, 
UK. Helen Lennox: Department of Neurology, NHS Tayside, Dundee, UK. Laura Marshall: Department of Neurology, NHS Forth Valley, Larbert, UK; Department of Neurology, NHS Fife, Kirkaldy, UK. Alison McEleney: Department of Clinical Neurosciences, NHS Lothian, Edinburgh, UK. Kitty Millar: Department of Neurology, NHS Highland, Inverness, UK. Suzanne Byrne: Department of Neurology, NHS Greater Glasgow \& Clyde, Glasgow, UK. Susan Stewart: Department of Neurology, NHS Tayside, Dundee, UK. Dorothy Storey: Department of Neurology, NHS Shetland, Shetland, UK. Gill Stott: Department of Clinical Neurosciences, NHS Lothian, Edinburgh, UK. Carol Thornton: Department of Neurology, NHS Grampian, Aberdeen, UK. Carolyn Webber: Department of Neurology, NHS Tayside, Dundee, UK.

Funding No funding was required for this study.

Data availability Upon request from the authors.

Conflicts of interest The authors declare that they have no competing interests.

Ethical standard statement Participants consented to inclusion in the CARE-MND register. Ethics approval for CARE-MND was provided by the Scotland A Research Ethics Committee (Approval: 15/SS/0126). Consent from patients was obtained at the time of their registration with the CARE-MND register.

\section{Reference}

1. World Health Organization (2020) WHO Coronavirus Disease (COVID-19) Dashboard. https://covid19.who.int/. Accessed 27 Aug 2020

2. Leighton D, Newton J, Colville S, Bethell A, Craig G, Cunningham L, Flett M, Fraser D, Hatrick J, Lennox H, Marshall L, McAleer D, McEleney A, Millar K, Silver A, Stephenson L, Stewart S, Storey D, Stott G, Thornton C, Webber C, Gordon H, Melchiorre G, Sherlock L, Beswick E, Buchanan D, Abrahams S, Bateman A, Preston J, Duncan C, Davenport R, Gorrie G, Morrison I, Swingler R, Chandran S, Pal S (2019) Clinical audit research and evaluation of motor neuron disease (CARE-MND): a national electronic platform for prospective, longitudinal monitoring of MND in Scotland. Amyotroph Lateral Scler Frontotemporal Degener 20(3-4):242-250. https://doi.org/10.1080/21678 421.2019.1582673

3. The Scottish Government (2016) Scottish Index of Multiple Deprivation (SIMD) 2016. Scottish Government. https://data.gov.uk/ dataset/a448dd2a-9197-4ea0-8357-c2c9b3c29591/scottish-index -of-multiple-deprivation-simd-2016. Accessed 15 Apr 2020 